Eur J Clin Chem Clin Biochem

1995; 33:727-732

(c) 1995 Walter de Gruyter \& Co.

Berlin · New York

\title{
Measurements of Haptoglobin by the Reaction with Concanavalin A in Sera of Patients with Ovarian Tumours ${ }^{1}$ )
}

\author{
By Iwona Katnik ${ }^{1}$, Joanna Jadach ${ }^{1}$, Krzysztof Kmieciak ${ }^{2}$, Jerzy Gerber ${ }^{2}$ and Wanda Dobryszycka \\ 1 Department of Chemistry \\ 2 Clinic of Gynaecology \\ ${ }^{3}$ Department of Biochemistry \\ Wroclaw University of Medicine, Wroclaw, Poland
}

(Received March 17/June 26, 1995)

Summary: The concentration of haptoglobin in sera of healthy women and patients with non-malignant and malignant ovarian tumours was measured by two methods, i.e. complex formation with haemoglobin and complex formation with concanavalin A. High correlation $(r=0.74)$ between both the methods was found in the group of healthy women, but correlation coefficients were much lower in the group of non-malignant and malignant tumours ( $r=0.42$ and 0.37 , respectively). Direct determinations of haptoglobin, and calculation of the ratio of haptoglobin bound to concanavalin A to haptoglobin bound to haemoglobin revealed statistically significant differences among the examined groups. Comparison of two methods of haptoglobin quantitation suggest that processes connected with ovarian disorders may alter the glycosylation of haptoglobin oligosaccharide chains.

\section{Introduction}

Haptoglobin is a genetically determined $\alpha_{2}$-acid glycoprotein, present in most body fluids. The oligosaccharide moiety of haptoglobin consists of $\mathrm{N}$-acetylglucosamine, mannose, galactose, fucose, and sialic acid, forming biand trianntennary $\mathrm{N}$-linked glycans. Haptoglobin is an "acute phase" reactant. Its plasma level is increased in malignancy, trauma, infection, inflammation, tissue damage, but decreased in haemolytic conditions, and since haptoglobin is synthesised in the liver (1) it is also decreased in severe hepatocellular deficiency.

Haptoglobin (Hp) is known to form with haemoglobin $(\mathrm{Hb})$ a stable, practically irreversible complex $(\mathrm{Hp}-\mathrm{Hb})$, which in vitro has the activity of a "true" peroxidase. This property is exploited for the quantitative determination of haptoglobin (2). Formation of the $\mathrm{Hp}-\mathrm{Hb}$ complex is a function of the polypeptide moiety of haptoglo-

\footnotetext{
1) This work was supported by the State Committee for Scientific Research grant No 6 P207 09004.
}

bin (1). On the other hand, the specific arrangement of carbohydrates in haptoglobin enables it to bind plant lectins. The reactivity of the mannose-type core of haptoglobin with concanavalin $\mathrm{A}$, which was utilized in the lectin-ELISA estimation of haptoglobin in body fluids, may change with alterations in haptoglobin glycosylation (3). Thus, changes in the measured concentrations of haptoglobin under various pathological conditions could be related to quantitative differences in hepatic haptoglobin synthesis, or/and to qualitative differences in the haptoglobin glycans.

Complex metabolic processes occuring in cancer include changes in structure and composition of the oligosaccharide chains of glycoproteins (increases/decreases in the content of specific carbohydrates, changes in antennary structure). These phenomena are very relevant clinically $(4,5)$.

In our previous paper haptoglobin determinations (by means of haemoglobin binding) in sera of patients with ovarian cancer were used to monitor the effects of chemotherapy (6). We also demonstrated some differences 
in the quantitation of haptoglobin in pathological body fluids, between the "traditional" $\mathrm{Hp}-\mathrm{Hb}$ method and the method based on Hp-concanavalin A complex formation (7). The purpose of the present study was to investigate whether these reactivities of haptoglobin, as determined in the sera of patients with ovarian tumours, might be used as an index of disease activity.

\section{Materials and Methods}

\section{Samples}

Control group (Group I) was established with sera from 37 healthy non-pregnant women (28-57 years old), attending medical followup in the Medical Diagnostic Centre in Wrocław. Serum samples were chosen for their normal haptoglobin values and on the basis of routine laboratory tests (erythrocyte sedimentation rate, activities of aminotransferases and acid phosphatase, glucose concentration etc.). Non-haemolysed serum samples were stored at $-20^{\circ} \mathrm{C}$ until analysed.

Pathological sera were collected from 99 women attending the Clinic of Gynaecology of Wroclaw University of Medicine. These preoperative patients were divided into two groups. Group II samples were taken from 56 women (25-61 years old) with non-malignant gynaecologic tumours (cysts, myomas). Sera from 43 patients ( $31-77$ years old), suffering from ovarian carcinomas (FIGO stages II-IV) were assigned to Group III. The staging was performed according to FIGO rules (8).

Quantification of haptoglobin based on its reactivity with haemoglobin

The Hp-Hb complex displays "true" peroxidase activity. The cleavage of hydrogen peroxide is indicated by guaiacol according to Jones \& Mould (2), as follows. Wells of the microtitration plate were cleaned and washed and used in place of small tubes. To the wells were added:

- $50 \mu \mathrm{l}$ of $\mathrm{Hp}$ standard $(5-300 \mathrm{mg} / \mathrm{l} 9 \mathrm{~g} / \mathrm{l} \mathrm{NaCl})$ or $5 \mu \mathrm{l}$ of serum sample in $50 \mu$ of $9 \mathrm{~g} / \mathrm{l} \mathrm{NaCl}$

$-50 \mu \mathrm{l} 0.5 \mathrm{~g} / \mathrm{l}$ horse methaemoglobin solution.

The control reagent sample containing all reagents and $50 \mu$ of saline instead of $\mathrm{Hp}$ was included in each series of estimations. A "blank" was prepared for each serum, containing the reagents and serum sample but without methaemoglobin. The plate was incubated for $10 \mathrm{~min}$ in room temperature, followed by addition of 150 $\mu \mathrm{l}$ of guaiacol reagent $(6.82 \mathrm{~g}$ guaiacol, $183 \mathrm{ml}$ of $1 \mathrm{~mol} / \mathrm{l}$ acetic acid added to $700 \mathrm{ml}$ distilled water, adjusted to $\mathrm{pH} \mathrm{4.0,} \mathrm{then} \mathrm{made}$ up to 1 litre with distilled water) and $50 \mu \mathrm{l}$ of $0.02 \mathrm{~mol} / \mathrm{l}$ hydrogen peroxide solution. After $5 \mathrm{~min}$, absorbance at $492 \mathrm{~nm}$ was measured in a Hamilton HR 7000 reader. Serum haptoglobin concentration was caluclated from a standard curve prepared for each plate.

Quantification of haptoglobin based on its reactivity with concanavalin A

The conditions of the assay for glycosylated haptoglobin bound to concanavalin A have been described in 1.c. (3). The lower detection limit of the assay calculated as 3 SD for the mean of the blank sample was $50 \mu \mathrm{g} / \mathrm{l}$. The intra-assay (within-day) and inter-assay (between day) coefficients of variation were $4-7 \%$ and $6-9 \%$, respectively, as analysed using sera with low, medium and high contents of haptoglobin bound to concanavalin A. The recovery of haptoglobin bound to concanavalin $\mathrm{A}$ in the presence of other glycoproteins was higher than $97 \%$.
The procedure of the assay was as follows: wells of the polystyrene microtitre plate were incubated at $37^{\circ} \mathrm{C}$ for $3 \mathrm{~h}$ with $0.1 \mathrm{~g} / \mathrm{l}$ concanavalin A in acetate buffer $0.1 \mathrm{~mol} / 1, \mathrm{pH} 6.0$, containing $1 \mathrm{mmol} / \mathrm{l}$ of each cation: $\mathrm{Ca}^{2+}, \mathrm{Mg}^{2+}$ and $\mathrm{Mn}^{2+}$. The plate was then washed 4 times with washing buffer $(0.005 \mathrm{~mol} / 1 \mathrm{HEPES}, \mathrm{pH}$ 7.2 , containing $0.15 \mathrm{~mol} / 1 \mathrm{NaCl}, 0.5 \mathrm{ml} / 1$ Tween $20,0.2 \mathrm{~g} / \mathrm{l}$ thiomersal and $1 \mathrm{mmol} / 1$ of divalent ions $\mathrm{Ca}^{2+}, \mathrm{Mg}^{2+}, \mathrm{Mn}^{2+}$ ). After blocking with $5 \mathrm{~g} / 1$ bovine serum albumih, $100 \mu \mathrm{l}$ of haptoglobin standard solutions (10-100 ng/well) and $100 \mu \mathrm{l}$ of diluted test samples (usual dilutions: 5000 and 10000 times) were added and incubated for $1 \mathrm{~h}$ at $37^{\circ} \mathrm{C}$. After washing 4 times with washing buffer containing $20 \mathrm{~g} / \mathrm{l}$ polyethylene glycol 6000 (PEG), $100 \mu \mathrm{l}$ of anti-Hp monoclonal antibody (clone 7.60.66.55) conjugated with peroxidase diluted (2000 times) with washing buffer (see above) was dispensed and the plate was incubated for $30 \mathrm{~min}$ at $37^{\circ} \mathrm{C}$. The plate was washed and the enzymatic activity was developed with $o$-phenylenediamine. The absorbance was read at $492 \mathrm{~nm}$ against a reference wavelength on a Hamilton UV HR 7000 ELISA reader.

Samples were analysed in triplicate after two dilutions to avoid an excess of glycoprotein. Controls were included for each experiment. Background absorbances (with buffer instead serum, but with all other reagents) ranged from 0.3 to 0.5 , depending on the plate and day of the experiment. Absorbance values for negative control (human serum without haptoglobin confirmed by Hp-Hb-ELISA (7)) were the same as background.

The amount of haptoglobin bound to concanavalin A was calculated from a standard curve prepared for each plate. The useful range of the standard curve was 50-600 $\mathrm{\mu g} / \mathrm{l}$. A standard preparation of haptoglobin containing $0.2 \%$ fucose, $2.6 \%$ mannose, $2.0 \%$ $\mathrm{N}$-acetylglucosamine, was used (9).

\section{Statistical calculations}

The average, median and standard deviation, lower and upper quartile, distribution of variables, correlation coefficient $r$ and probability level among the examined groups (Wilcoxon test) were calculated.

\section{Results}

Haptoglobin concentration was estimated in sera of patients with non-malignant (Group II) and malignant (Group III) ovarian tumours as compared with control healthy women (Group I). The determinations were carried out in parallel, using two methods i. e. complex formation with $\mathrm{Hb}$ and complex formation with concanavalin A (fig. 1). Correlation coefficients for both methods were: in the control group $r=0.74$, in the group of non-malignant tumours $r=0.42$, and in the cancer group $r=0.37$.

Table 1 summarises the statistical data of the Hp-concanavalin $\mathrm{A}$ and $\mathrm{Hp}-\mathrm{Hb}$ determinations. The ratio of $\mathrm{Hp}-$ concanavalin $\mathrm{A}$ to $\mathrm{Hp}-\mathrm{Hb}$ was also calculated. In the control group, the three measured values are rather similar (approximately 1). In the group of non-malignant tumours the mean value for $\mathrm{Hp}-\mathrm{Hb}$ was twice as high as in the control group, while Hp-concanavalin A was only a little higher. In the group of ovarian cancers $\mathrm{Hp}-\mathrm{Hb}$ was more than three times and Hp-concanavalin A more than four times higher than in theicontrol group. In both 

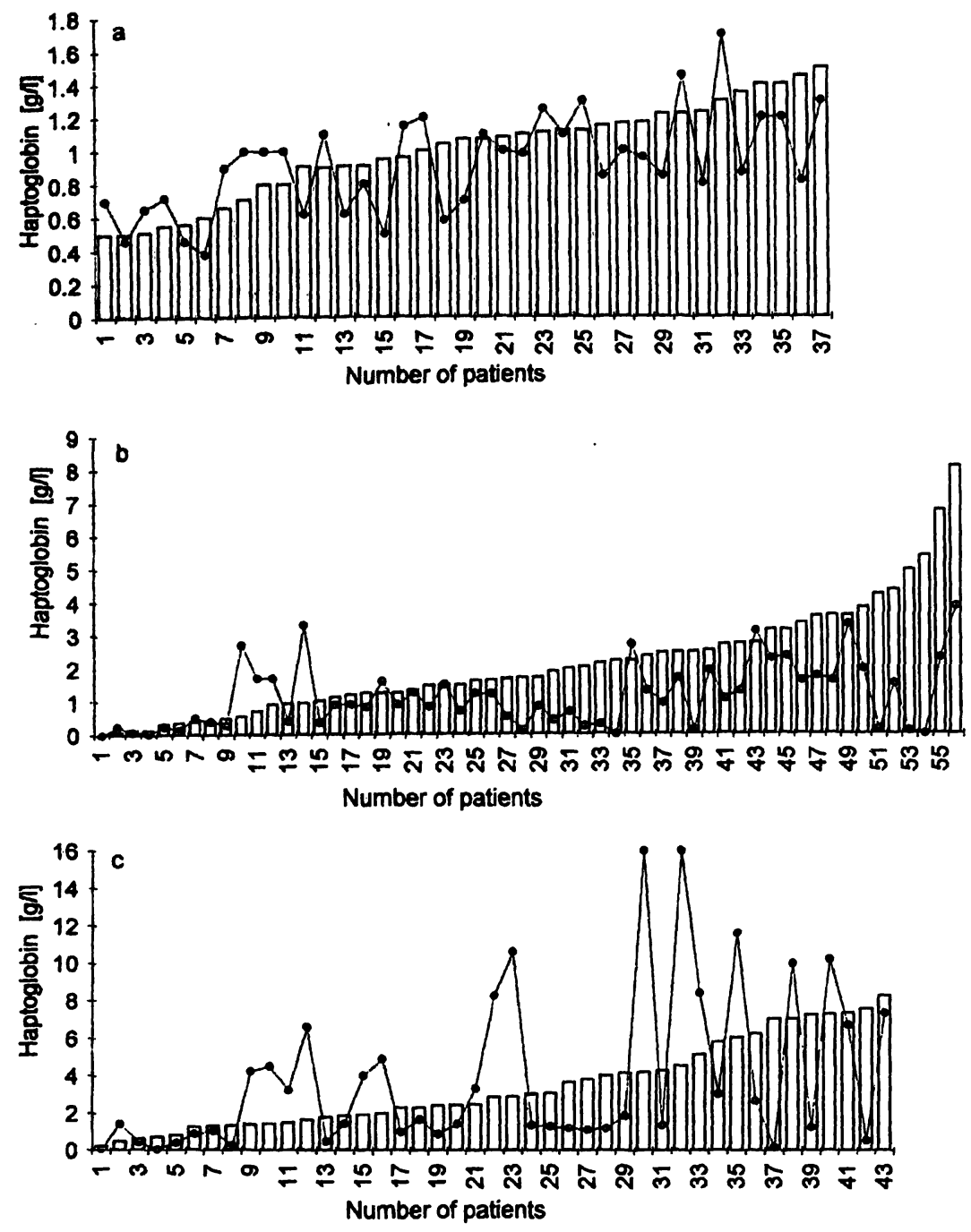

Fig. 1 Comparison of haptoglobin concentrations measured by the reaction with haemoglobin (empty bars) and with concanavalin
A (black squares) in healthy women (a), patients with non-malignant tumours (b) and with ovarian cancers (c). pathological groups the mean values of the ratio were 0.83 and 1.24 , with similar high SD values caused by extreme variation of individual results (fig. 1).

The results were analysed by means of the Pearson $\left(\chi^{2}\right)$ and Wilcoxon tests at $\mathrm{p}=0.05$. The values for $\mathrm{Hp}$ in the control group showed a normal distribution, whereas the distribution was not normal in the other two groups. The following statistically significant differences were found in:

Hp-Hb level - Group I vs. Group II and Group III; Group II vs. Group III.

Hp-ConA - Group I vs. Group III; level Group II vs. Group III.

Ratio - Group I vs. Groups III; Group II vs. Group III.

Individual data (fig. 1) as well as statistical analysis (tab. 1) show relatively wide distribution of results in both pathological groups. In order to visualize "normal", higher and lower values of $\mathrm{Hp}-\mathrm{Hb}, \mathrm{Hp}$-concanavalin A and ratio in these groups, we established certain limits (means $\pm 2 \mathrm{SD}$ ) based on the measurements in the control group. There were: $\mathrm{Hp}-\mathrm{Hb}=0.44-1.56 \mathrm{~g} / \mathrm{l}, \mathrm{Hp}$ concanavalin $\mathrm{A}=0.35-1.51 \mathrm{~g} / \mathrm{l}$, ratio $=0.45-1.49$.

As can be seen in figure 2, the majority of $\mathrm{Hp}-\mathrm{Hb}$ and $\mathrm{Hp}$-concanavalin A determinations in the pathological groups remained outside the limits (more than $70 \%$ and about $60 \%$, respectively). Differences in $\mathrm{Hp}-\mathrm{Hb}$ and $\mathrm{Hp}$ concanavalin A measurements are also apparent in the ratio. Thus, in the group with non-malignant tumours $10 \%$ of the ratio values were higher, $30 \%$ lower and $60 \%$ normal, whereas in the cancer group $30 \%$ of the respective results were higher, $40 \%$ lower and only $30 \%$ "normal".

It is worth pointing out that the ratio in pathological groups was "normal" in some cases when the $\mathrm{Hp}-\mathrm{Hb}$ was abnormal or was "off-limits" when $\mathrm{Hp}-\mathrm{Hb}$ was "normal". For instance $\mathrm{Hp}-\mathrm{Hb}$ and $\mathrm{Hp}$-concanavalin A concentrations in a patient with ovarian cancer were extremely high: $6.7 \mathrm{~g} / 1$ and $7.3 \mathrm{~g} / 1$, respectively, hence the 
ratio (=0.92) was "normal". Another case in which both $\mathrm{Hp}$ values were abnormally low (approximately 0.3 $\mathrm{g} / \mathrm{l})$ the ratio $(=1.0)$ was "normal". Perhaps more interesting are those cases where both determinations are incompatible. For instance, $\mathrm{Hp}-\mathrm{Hb}$ values of $1.65,1.02$ and $1.39 \mathrm{~g} / \mathrm{l}, \mathrm{Hp}$-concanavalin $\mathrm{A}$ values of $3.5,0.3$ and $4.25 \mathrm{~g} / \mathrm{l}$, resulting in ratios of $2.12,0.29$ and 3.06 , respectively.

\section{Discussion}

Of all gynaecologic malignancies ovarian cancer has the lowest overall survival rate. Most patients have advanced stage disease at the time of diagnosis. Monitoring and detection of subclinical disease is limited because the majority of patients have no measurable disease. Many biochemical and immunological quantities have been used, but none has fulfilled the criteria of an ideal tumour marker $(10-13)$. Although failing to meet such criteria, haptoglobin measurements have been useful in assessing the disease status of ovarian cancer patients, particularly during chemotherapy, and have helped to decide on surgery or a "second-look" operation $(6,14)$.

Tab. 1 Haptoglobin (Hp) levels estimated from haemoglobin $(\mathrm{Hb})$ and concanavalin $\mathrm{A}$ (ConA) reactivities in sera of patients with ovarian tumours.

\begin{tabular}{llll}
\hline Groups & $\mathrm{Hp}-\mathrm{Hb}$ & $\mathrm{Hp}-\mathrm{ConA}$ & Ratio \\
$\mathrm{g} / \mathrm{l}$ & $\mathrm{g} / \mathrm{l}$ & $\begin{array}{l}\mathrm{Hp}-\mathrm{Con} \mathrm{A} \\
\mathrm{Hp}-\mathrm{Hb}\end{array}$ \\
\hline
\end{tabular}

$\begin{array}{llll}\begin{array}{l}\text { I. Normal sera } \\ \mathrm{n}=37\end{array} & & & \\ \text { Mean value } \pm \mathrm{SD} & 1.0 \pm 0.28 & 0.93 \pm 0.64 & 0.97 \pm 0.26 \\ \text { Median } & 1.07 & 0.96 & 0.91 \\ \text { Range of values } & 0.5-1.50 & 0.38-1.7 & 0.55-1.4 \\ \text { Lower quartile } & 0.8 & 0.7 & 0.74 \\ \text { Upper quartile } & 1.17 & 1.1 & 1.2\end{array}$

II. Non-malignant tumours $\mathrm{n}=56$

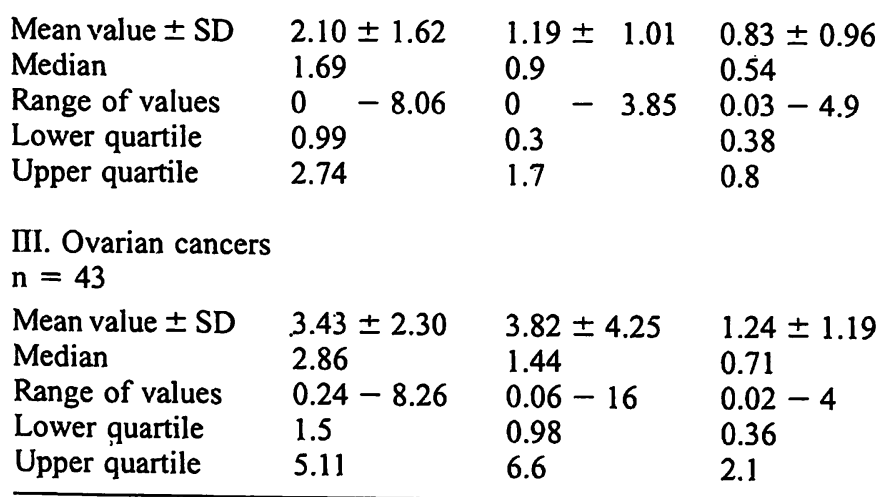

$\mathrm{Hp}-\mathrm{Hb} \quad-$ haptoglobin level measured by the reaction with haemoglobin (2).

Hp-ConA - haptoglobin level measured by the reaction with SD concanavalin A (3).

- standard deviation
It is well documented that quantitative changes in the concentrations of acute phase proteins during certain pathophysiological states, particularly in inflammatory conditions and cancer, often accompany specific alterations of the carbohydrate moieties of glycoproteins. Changes in the degree of branching (major microheterogeneity) or/and in terminal glycosylation of glycoproteins (minor microheterogeneity) originate from modifications in the post-translational glycosylation mechanism in the liver or from degradative processes in the circulation $(4,5,15,16)$.

The introduction of affinity chromatography and crossed affinity electrophoresis of haptoglobin with plant lectins (concanavalin A and wheat germ agglutinin), leading to the appearance of several microheterogeneous forms, was shown to be of value in the differential diagnosis of ovarian cancer and other inflammatory conditions. The carbohydrate moiety of haptoglobin from ascitic fluids of patients with ovarian carcinoma was apparently changed as judged from the reactions with haemoglobin and concanavalin A $(17,18)$. Investigations of ovarian cancer haptoglobins using digoxigenin-labelled lectins suggest that normal and ovarian cancer haptoglobins differ in their content of carbohydrate structures containing sialic acid-linked $\alpha(2-6)$ or $\alpha(2-3)$ to galactose and in the type of glycan branching (9). Turner's group (1922) has shown that ovarian cancer sera have high levels of an abnormal form of haptoglobin in which fucose occupies three positions (mainly $\alpha(1-3)$ ) in carbohydrate chains. The fucose content of haptoglobin was elevated 7-fold in ovarian cancer. The increased fucosylation of haptoglobin was closely related to tumour burden and also correlated with the serum levels of $\alpha$-3-fucosyl transferase. Analyses of galactose and $\mathrm{N}$-acetylglucosamine contents of haptoglobin suggest that there are more oligosaccharide branches on the "cancer haptoglobin".

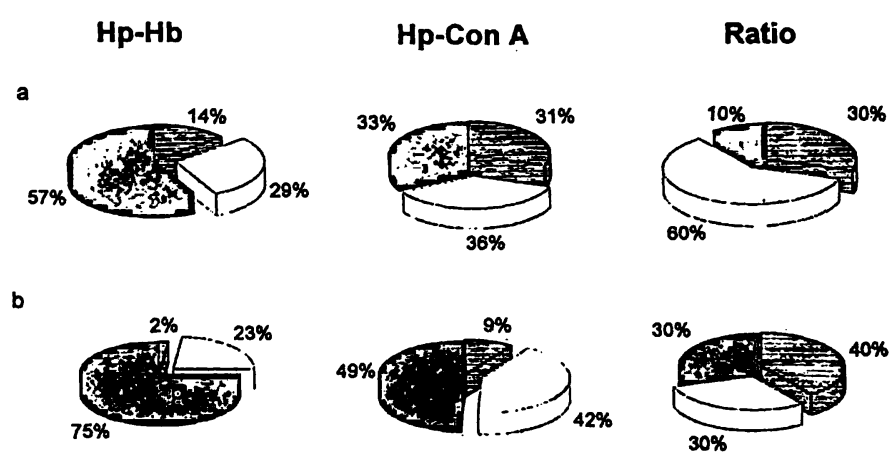

Fig. 2 Distribution of haptoglobin concentrations in patient sera with non-malignant tumours (a) and ovarian cancers (b).

Results are expressed as a percentage of $\square$ "normal", $a$ higher, and $\square$ lower Hp measurements at established limits (means \pm 2 SD). The distribution of values in the pathological groups was related to the limits established for the control group: $\mathrm{Hp}-\mathrm{Hb}=0.44-1.56$ $\mathrm{g} / \mathrm{l}$; Hp-concanavalin $\mathrm{A}=0.35-1.51 \mathrm{~g} / 1$,'ratio $=0.45-1.49$. 
The present work shows the amount of haptoglobin that can react with concanavalin A in sera from women with ovarian tumours. We compared these levels with haptoglobin concentrations as measured by the traditional haptoglobin-haemoglobin peroxidase method (2). Comparisons were expressed as ratio values of Hp-concanavalin $\mathrm{A}$ to $\mathrm{Hp}-\mathrm{Hb}$. The lectin concanavalin $\mathrm{A}$ binds with bi-, but not tri- or tetraantennary $\mathrm{N}$-linked glycans, but the presence of an additional bisecting GlcNAc residue on the Man of the biantennary units inhibits this binding. The degree of reactivity with concanavalin A depends on the number of biantennary structures present on the molecule and could help to reveal major microheterogeneity in glycoproteins.

Measurements of $\mathrm{Hp}$-concanavalin A and $\mathrm{Hp}-\mathrm{Hb}$ in the present paper provide interesting data on the differential diagnosis of malignant against non-malignant ovarian tumours, as well as non-malignant tumours against other physiological states (see statistically significant differences). In both pathological groups, about $60 \%$ of results were "off limits" (fig. 2). Moreover some differences in Hp-concanavalin A concentrations between non-malignant and malignant groups were observed, namely, $31 \%$ and $33 \%$ of results in non-malignant vs. 9 and $49 \%$ in malignant sera were lower and higher than "normal", respectively. In spite of the above mentioned facts, we think that the comparison of Hp-concanavalin A with $\mathrm{Hp}-\mathrm{Hb}$ estimations (i. e. calculation of the ratio) could give valuable information on the degree of glycosylation of $\mathrm{Hp}$ present in the blood of patients. During ovarian disorders, haptoglobin, an acute phase protein, is increased 2-5-fold, as measured by its reaction with haemoglobin. The results of Hp-concanavalin A and Hp$\mathrm{Hb}$ measurements in all the patients with ovarian tumours fall into three subgroups.

First subgroup: no differences in Hp-concanavalin $\mathrm{A}$ and $\mathrm{Hp}-\mathrm{Hb}$ measurements were found (ratio $0.97 \pm 0.52$ ), possibly signifying that no differences in the degree of branching of haptoglobin glycans were detected and that the antennary structure of haptoglobin glycans in these patients was similar to that present in healthy women. Sixty percent of the ratio in non-malignant group and $30 \%$ in ovarian cancer were "normal".

\section{References}

1. Dobryszyoka W. Haptoglobin: retrospectives and perspectives. In: Mackiewicz A, Kushner I, Baumann H, editors. Acute phase proteins: molecular biology, biochemistry, and clinical applications. Boca Raton, Ann Arbor, London, Tokyo: CRC Press, 1993:185-206.

2. Jones GE, Mould DL. Adaptation of the guaiacol (peroxidase) test for haptoglobins to a microtitration plate system. Res Vet Sci $1984 ; 37: 87-92$.
Second subgroup: the Hp-concanavalin A level was significantly higher than the $\mathrm{Hp}-\mathrm{Hb}$ level (ratio higher than 1.5); this was observed in 10 and $30 \%$ of analysed samples in non-malignant and cancer disease, respectively. These samples probably contained increased amount of haptoglobin glycoforms with biantennary glycans.

Third subgroup: the Hp-concanavalin A level was lower than the $\mathrm{Hp}-\mathrm{Hb}$ (ratio lower than 0.45). This kind of pattern was noticed in $30 \%$ and $40 \%$ of data in nonmalignant and cancer disease, respectively. It is predicted that these samples are rich in tri- or higher antennary haptoglobin glycans, or biantennary glycans containing an additional bisecting $\mathrm{N}$-acetylglucosamine.

Mackiewicz \& Kushner (15) have postulated that changes in the glycosylation patterns of acute phase proteins in patient sera during acute inflammation differ from those seen in chronic inflammation. During acute inflammation glycoproteins are characterized by higher amounts of biantennary glycans with increased concanavalin A reactivity. In contrast, in chronic inflammation, a relative increase of tetra- and/or triantennary structures with decreased concanavalin A reactivity were found.

It appears that the haptoglobin of some patients with ovarian tumours (second subgroup) had a higher content of biantennary glycans, while in others (third subgroup) there was an increase of tetra- and triantennary structures. In contrast, the high fucose content in haptoglobin from ovarian cancer patients is known to be branchingindependent $(19,21)$.

The results reported here are the starting point for two further lines of study, namely oncological-gynaecological diagnostics and the study of the structure and arrangement of carbohydrates of glycoproteins synthesized in the human organism under the influence of malignancy.

Last but not least we would like to point out that estimations of $\mathrm{Hp}$-concanavalin $\mathrm{A}$ and $\mathrm{Hp}-\mathrm{Hb}$ are rapidly and easily performed and not expensive, making them suitable for use in routine laboratory practice.

3. Katnik I, Dobryszycka W. Development of concanavalin Aenzyme immunosorbent assay for glycated haptoglobin using polyclonal and monoclonal antibodies. J Immunoassay 1992; 13:145-62.

4. Van Dijk W, Turner GA, Mackiewicz A. Changes in glycosylation of acute-phase proteins in health and disease: occurence, regulation and function. Glycosylation and Disease 1994; 1:15-9. 
5. Turner GA. N-glycosylation of serum proteins in disease and its investigation using lectins. Clin Chim Acta 1992; 208:149-71.

6. Dobryszycka W, Gerber J, Zuwala-Jagiełło J, Ujec M. Acute phase reactants and circulating immune complexes in patients with ovarian carcinoma. Arch Immunol Ther Exp 1991; $39: 41-50$.

7. Kątnik I, Dobryszycka W. Enzyme immunoassay to measure low levels of haptoglobin in biological fluids. J Immunoassay 1990; 11:503-17.

8. Kottmeier HL. Report presented by the Cancer Committee to the General Assembly of FIGO 1971; 9:178-9.

9. Hempling RE. Tumor markers in epithelial ovarian cancer. Clinical applications. Obstet Gynecol Clinics North America 1994; $21: 41-61$.

10. Iwanari O, Miyako J, Date Y, Nakayama S, Kijima S, Moriyama $M$, et al. Differential diagnosis of ovarian cancer, benign ovarian tumor and endometriosis by a combination assay of serum sialyl SSEA-1 antigen and CA 125 levels. Gynecol Obstet Invest 1990; 29:71-4.

11. Bates SE. Clinical applications of serum tumor markers. Am Intern Med 1991; 115: 623-38.

12. Einhorn N, Knapp RC, Bast RC, Zurawski VR. CA 125 assay used in conjunction with CA 15-3 and TAG-72 assays for discrimination between malignant and non-malignant diseases of the ovary. Acta Oncologica 1989; 28:655-7.

13. Warwas M, Gerber J, Pietkiewicz A. Haptoglobin and proteinase inhibitors in the blood serum of women with inflammatory, benign and neoplastic lesions of the ovary. Neoplasma 1981; 33:79-84.

14. Kushner I, Mackiewicz A. The acute phase response: an overview. In: Mackiewicz A, Kushner I, Baumann H, editors. Acute phase proteins molecular biology, biochemistry, and clinical applications. Boca Raton, Ann Arbor, London, Tokyo: CRC Press, 1993: 3-19.
15. Taketa $\mathrm{K}$, Hirai $\mathrm{H}$. Lectin affinity electrophoresis of $\alpha$-fetoprotein in cancer diagnosis. Electrophoresis 1989; 10:562-7.

16. Katnik I. Studies on haptoglobin binding to concanavalin A. Biochim Biophys Acta 1984; 790:8-14.

17. Katnik I, Gerber J, Dobryszycka W. Affinoimmunoelectrophoresis of haptoglobin with Wheat Germ Agglutinin. Diagnostic significance in ovarian carcinoma. Arch Immunol Ther Exp 1987; 35:547-52.

18. Katnik I, Jadach J, Krotkiewski $\mathrm{H}$, Gerber J. Investigating the glycosylation of normal and ovarian cancer haptoglobins using digoxigenin-labelled lectins. Glycosylation and Disease 1994; 1:97-104.

19. Thompson S, Dargan E, Turner GA. Increased fucosylation and other carbohydrate changes in haptoglobin in ovarian cancer. Cancer Lett 1992; 66:43-8.

20. Thompson S, Turner GA. Elevated levels of abnormally-fucosylated haptoglobins in cancer sera. $\mathrm{Br} \mathrm{J}$ Cancer 1987; $56: 605-10$.

21. Dargan E, Thompson S, Cantwell J, Wilson RG, Turner GA. Changes in the fucose content of haptoglobin in breast and ovarian cancer: association with disease progression. Glycosylation and Disease 1994; 1:37-43.

22. Thompson S, Cantwell BMJ, Matta KL, Turner GA. Parallel changes in the blood levels of abnormally-fucosylated haptoglobin and alpha 1,3 fucosyltransferase in relationship to tumour burden: more evidence for a disturbance of fucose metabolism in cancer. Cancer Lett 1992; 65:115-21.
Dr Iwona Kątnik
Bujwida 44a
PL-50-345 Wrocław
Poland

Department of Chemistry

Wrocław University of Medicine 